\title{
Correction to: Conducting an observational study during an economic crisis: analysis of the treatment and follow-up phase of Greek patients participating in the ExFOS study
}

\author{
Kyriakos Aloumanis ${ }^{1} \cdot$ G. Kapetanos $^{2} \cdot$ T. Alexandridis ${ }^{3} \cdot$ V. Drossinos ${ }^{1} \cdot$ N. Papaioannou ${ }^{4} \cdot$ for the Greek ExFOS study \\ group
}

Published online: 11 March 2019

(C) Hellenic Endocrine Society 2019

\section{Correction to: Hormones}

https://doi.org/10.1007/s42000-018-0077-6

Unfortunately in the original publication, the affiliation of the author N. Papaioannou was incorrectly provided.

The correct affiliation for N. Papaioannou should read as follows:

4 Laboratory for the Research of Musculoskeletal System, School of Medicine, National and Kapodistrian University of Athens, Greece

Publisher's note Springer Nature remains neutral with regard to jurisdictional claims in published maps and institutional affiliations.

The online version of the original article can be found at https://doi.org/ 10.1007/s42000-018-0077-6

Kyriakos Aloumanis

aloumanis_kyriakos@lilly.com

1 Department of Medical Research, Pharmaserve Lilly, 15th Km National Road Athens-Lamia, Kifissia, Greece

2 Papageorgiou General Hospital, 3rd Orthopedics University Clinic, Thessaloniki, Greece

3 Endocrine Division, Department of Medicine, Patras University Hospital, Patras, Greece

4 Laboratory for the Research of Musculoskeletal System, School of Medicine, National and Kapodistrian University of Athens,

Athens, Greece 